\title{
A mechanical explanation of RNA pseudoknot function in programmed ribosomal frameshifting
}

\author{
Olivier Namy ${ }^{1 \star} \uparrow$, Stephen J. Moran ${ }^{2 \star}$, David I. Stuart ${ }^{2,3}$, Robert J. C. Gilbert ${ }^{2,3} \&$ lan Brierley ${ }^{1}$
}

The triplet-based genetic code requires that translating ribosomes maintain the reading frame of a messenger RNA faithfully to ensure correct protein synthesis ${ }^{1}$. However, in programmed -1 ribosomal frameshifting ${ }^{2}$, a specific subversion of frame maintenance takes place, wherein the ribosome is forced to shift one nucleotide backwards into an overlapping reading frame and to translate an entirely new sequence of amino acids. This process is indispensable in the replication of numerous viral pathogens, including HIV and the coronavirus associated with severe acute respiratory syndrome ${ }^{3}$, and is also exploited in the expression of several cellular genes ${ }^{4}$. Frameshifting is promoted by an mRNA signal composed of two essential elements: a heptanucleotide 'slippery' sequence ${ }^{5}$ and an adjacent mRNA secondary structure, most often an mRNA pseudoknot ${ }^{6}$. How these components operate together to manipulate the ribosome is unknown. Here we describe the observation of a ribosome-mRNA pseudoknot complex that is stalled in the process of -1 frameshifting. Cryoelectron microscopic imaging of purified mammalian $80 \mathrm{~S}$ ribosomes from rabbit reticulocytes paused at a coronavirus pseudoknot reveals an intermediate of the frameshifting process. From this it can be seen how the pseudoknot interacts with the ribosome to block the mRNA entrance channel, compromising the translocation process and leading to a spring-like deformation of the P-site transfer RNA. In addition, we identify movements of the likely eukaryotic ribosomal helicase and confirm a direct interaction between the translocase eEF2 and the P-site tRNA. Together, the structural changes provide a mechanical explanation of how the pseudoknot manipulates the ribosome into a different reading frame.

Programmed -1 ribosomal frameshifting was first described in 1985 (ref. 7) but the mechanism of the process has remained elusive. Subsequently ${ }^{8-10}$ it was shown that RNA pseudoknot structures pause ribosomes over a homopolymeric slippery sequence, at which the ribosome-bound tRNAs realign in the -1 frame $^{5}$, but that pausing alone is insufficient to promote frameshifting ${ }^{8-11}$. To gain insight into this mechanism at the molecular level, we developed a method of purifying rabbit reticulocyte lysate (RRL) ribosomes stalled in the act of decoding a frameshift signal and subjected them to cryoelectron microscopy (cryo-EM) analysis (Methods and Supplementary Fig. 1). Stalled complexes were assembled on a short mRNA transcript containing a variant of the coronavirus IBV frameshift signal ${ }^{6}$ in which the slippery sequence was replaced by a sequence that is unslippery $^{12}$ (CGAGGCA). Such complexes have been shown to represent true intermediates, and not 'dead-end' products ${ }^{9-11}$. Three reconstructions of RRL ribosomes were produced. The first was the translating $80 \mathrm{~S}$ ribosome, stalled at the IBV pseudoknot (PK) and containing densities consistent with the presence of a P-site
tRNA and elongation factor 2 (eEF2) as judged from modelling and comparative analysis $\left(80 \mathrm{~S}_{\mathrm{PK}}\right.$; Fig. 1a). The resolution of this reconstruction was $16.2 \AA$ (see Supplementary Information), for which 12,161 out of a total of 17,672 images were selected as being stalled with full and equal occupancy of eEF2, tRNA and pseudoknot. Structural changes associated with pseudoknot-induced -1 frameshifting were then determined by reference to the two control reconstructions. The first of these was an apo-80S ribosome $\left(80 \mathrm{~S}_{\mathrm{Apo}}\right.$; Fig. 1b), incorporating 10,296 images with a resolution of $14 \AA$. The second was created by deletion of the pseudoknot loops and connection of the two stems into a single stem-loop (SL; Supplementary Fig. 1) to form a structure capable of stalling the ribosome sufficiently to allow purification but leaving it unable to promote efficient frameshifting ${ }^{11}\left(80 \mathrm{~S}_{\mathrm{SL}}\right.$; Fig. 1c). The $80 \mathrm{~S}_{\mathrm{SL}}$ reconstruction resolution was $15.7 \AA$, for which 9,805 out of 14,887 images were selected as being stalled with tRNA bound. The ribosomes purified with this stem-loop were treated and prepared in exactly the same way as the pseudoknot-engaged ribosome. Any differences observed between these two reconstructions, such as bound cofactors, can therefore be ascribed directly to the action of the functional pseudoknot.

Our reconstructions reveal that the pseudoknot interacts with the ribosome at the entrance to the mRNA channel, in close association with a likely mammalian 80 S helicase $^{13}$ (Fig. 2, left panel). This confirms previous suggestions ${ }^{13,14}$ of the possible position of the pseudoknot structure. From the path taken by a short synthetic mRNA through the 70S ribosome decoding site, it has been estimated that mRNA enters the ribosome around nucleotides +13 to +15 (where the first residue of the P-site AUG codon is designated +1 (ref. 14)). In our mRNA the first base of the pseudoknot was at position +14 with respect to the first base of the P-site and thus would be expected to be located at the entrance to the mRNA tunnel as we see it in our map. It has also been shown ${ }^{13}$ that the $70 \mathrm{~S}$ ribosome can itself act as a helicase for unwinding mRNA secondary structures, with the active site at position +11 , located between the head and shoulder of the 30S subunit. Prokaryotic ribosomal proteins S3, S4 and S5 that line the entrance to the tunnel are implicated in helicase activity; they potentially form a ring around the incoming mRNA, acting as a processivity clamp. Superimposition of our pseudoknot-stalled and control maps allows a visual representation of differences between the maps found in this region (Fig. 2). It has been suggested that the mode of action of the pseudoknot arises from stereochemical mismatch between the ribosomal helicase and the pseudoknot structure $^{14}$. In our pseudoknot-engaged map, three regions of density surrounding the mRNA entrance tunnel seem to move up and contact the putative pseudoknot structure (Fig. 2, left panel). A yeast mode ${ }^{15}$ identifies one of these regions as rpS3 (in prokaryotes, 
S3), one as $18 \mathrm{~S}$ rRNA helix 16, which interacts with rpS9 (S4 in prokaryotes), and a final region as rpS2 (S5 in prokaryotes). In addition, the ubiquitous eukaryotic ribosomal regulatory protein RACK1 undergoes a movement that seems connected to the presence of the pseudoknot. This observation represents the first view of a ribosomal helicase in action. Futhermore, the interaction of the pseudoknot with the mammalian equivalents of S3, S4 and S5 indicates that part of the pseudoknot's functional significance for the elongating ribosome is indeed an interaction with the ribosomal helicase. Resistance of the pseudoknot to the action of the helicase may contribute to the stability of the overall complex, trapping the elongating ribosome.

The $80 \mathrm{~S}_{\mathrm{PK}}$ complex shows a ratchet-like rearrangement of the ribosome linked to the trapping of eEF2 (refs 16, 17). No antibiotics were used to trap eEF2 (as were used in previous studies), indicating that such a structure might indeed be physiological ${ }^{18}$. As neither eEF2 nor a ratchet-like rotation is observed in the $80 \mathrm{~S}_{\mathrm{SL}}$ complex, in the $80 \mathrm{~S}_{\mathrm{PK}}$, the changes can be attributed to the functional pseudoknot structure. These features indicate that the $80 \mathrm{~S}_{\mathrm{PK}}$ ribosome has been stalled by the pseudoknot during translocation, with eEF2 precluding the binding of a tRNA in the A-site until the frameshift has been completed. This first visualization of an elongating eukaryotic ribosome stalled in the act of translocation confirms a direct interaction between eEF2, the translocase, and the P-site tRNA. We can therefore identify translocation as the point in the elongation cycle at which frameshifting occurs, as has been suggested previously ${ }^{19,20}$. This interplay of pseudoknot, tRNA, eEF2 and structural rearrangement of the ribosome directly illustrates the dynamic and concerted nature of the frameshifting process.

The P-site tRNA in the pseudoknot-engaged reconstruction is distorted in comparison with the stem-loop reconstruction (Fig. 2, right panel) such that the anticodon is raised by a bending of the tRNA towards the A-site occupied by the tip of eEF2. Although the shape of the T-arm and acceptor stem remains unmodified, the elbow of the tRNA is pushed upwards towards the roof of the P-site (against the large subunit), indicating the presence of opposing forces. The present resolution $(16 \AA)$ is insufficient to permit the visualization of atomic details; however, it seems that the conformational change in the tRNA derives essentially from a bending of the D-arm (Fig. 2,
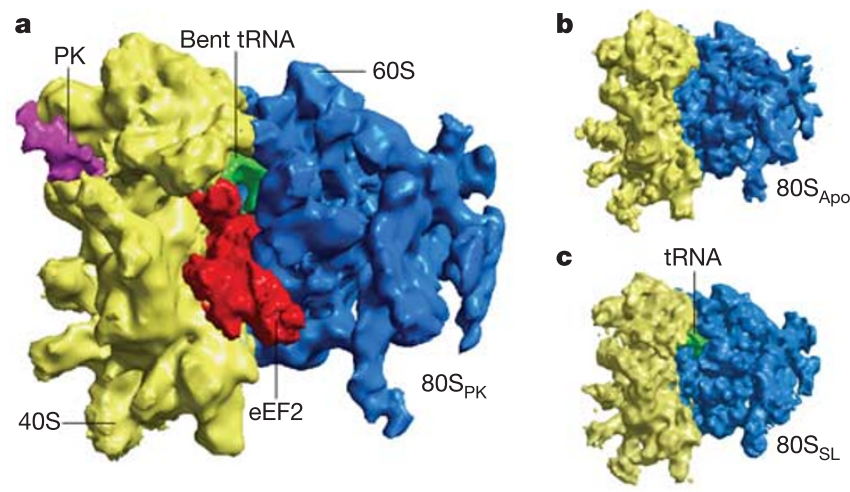

Figure 1 Structures of stalled $80 \mathrm{~S}$ complexes. a, The pseudoknot-engaged rabbit $80 \mathrm{~S}$ ribosome $\left(80 \mathrm{~S}_{\mathrm{PK}}\right)$ viewed in profile. The large $60 \mathrm{~S}$ subunit is coloured blue and the small $40 \mathrm{~S}$ subunit is coloured yellow. The P-site tRNA stalled within the complex is coloured green, the eukaryotic translocase (eEF2) red and the pseudoknot structure (PK) purple. $\mathbf{b}$, The control apo$80 \mathrm{~S}$ ribosome $\left(80 \mathrm{~S}_{\mathrm{Apo}}\right)$ with the subunits coloured as in b. $\mathrm{c}$, The structure of a ribosome purified in the same way as $80 \mathrm{~S}_{\mathrm{PK}}$, but with the pseudoknot modified to form a stem-loop with greatly reduced capacity to induce frameshifting $\left(80 \mathrm{~S}_{\mathrm{SL}}\right)$. The subunits are coloured as in $\mathbf{b}$ and $\mathbf{c}$; the P-site tRNA stalled within the complex is coloured green. right panel). The distortion of the P-site tRNA in these complexes is similar to the flexibility of the amino-acyl tRNA previously observed during the accommodation process ${ }^{21}$, in which the processes of codon-anticodon interaction and accommodation of cognate tRNAs are viewed ${ }^{21}$ as a dynamic interplay involving the mechanical properties of tRNA as well as those of the ribosome. The similar mechanical deformation observed in our pseudoknot-stalled complex indicates that the spring-like properties of tRNA might also be important in frameshifting.

The insight into the frameshifting process presented here leads us to propose a mechanical explanation for programmed -1 frameshifting (Fig. 3). When the elongating ribosome encounters the pseudoknot structure, the helicase action at the mRNA entrance tunnel attempts to unwind the pseudoknot but stereochemical mismatch prevents it from doing so. During translocation, the movement of tRNA through the ribosome is resisted by tension developed in the mRNA strand by the pseudoknot blockage at the tunnel entrance (as suggested previously ${ }^{22}$ ). The mRNA in turn is connected to the tRNA by means of the codon-anticodon interaction. Because the tRNA is prevented from returning to the A-site by the presence of eEF2, the ribosome, in attempting to translocate the anticodon into the authentic P-site, places strain on the tRNA that results in the adoption of a bent conformation. The opposing actions of translocation, catalysed by eEF2, and pulling from the mRNA strand account for the bending of the tRNA, spring-like, in a $(+)$ sense ( $3^{\prime}$ direction) and the movement of the elbow of the tRNA into the roof of the P-site. These opposing forces place a strain on the codon-anticodon interaction that promotes breakage. Subsequent relaxation of the bent tRNA structure would then be in a $(-)$ sense

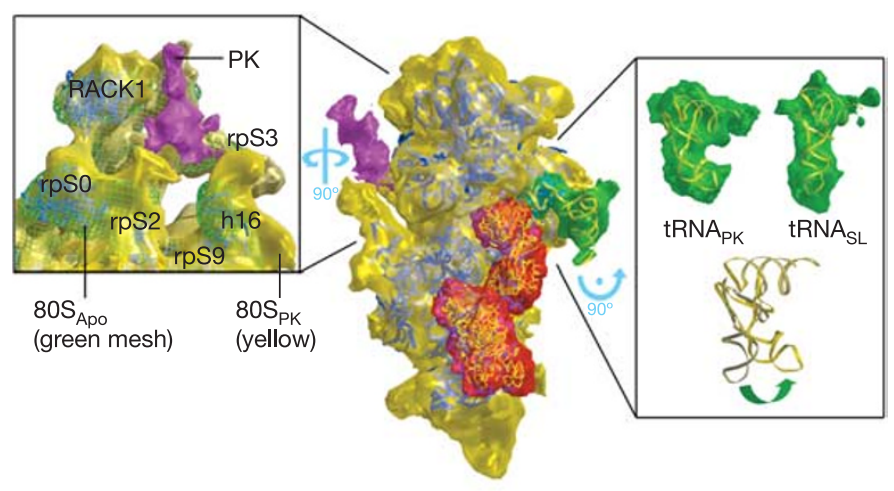

Figure 2 Atomic fits to the small subunit and bound cofactors of the pseudoknot-engaged ribosome. In the centre the small subunit is viewed in a similar orientation to that of Fig. 1, with the large subunit removed computationally. The subunit, tRNA, eEF2 and pseudoknot are coloured as in Fig. 1. The yeast atomic model for the small subunit ${ }^{15}$ has been fitted to the subunit itself (blue ribbons), the structure of eEF2 (ref. 18) to the corresponding density (yellow coil), and the tRNA stalled within the complex likewise (yellow ribbon). A stereo view of the central image is provided in Supplementary Fig. 2. Left and right: close-up views, rotated as indicated to afford a detailed picture of the structural changes associated with the engaged pseudoknot (left) and the stalled tRNA (right). Left: a view from the solvent face of the $80 S_{\mathrm{PK}}$ small subunit (yellow), with the $80 \mathrm{~S}_{\mathrm{Apo}}$ structure (green mesh) superimposed. Movements of the subunit in the $80 S_{\mathrm{PK}}$ ribosome up and towards the pseudoknot structure relative to the $80 S_{\text {Apo }}$ ribosome can be seen. Here the atomic fits (blue ribbon) are to the $8_{00} S_{\text {Apo }}$ structure. From this fitting the movements can be associated with the eukaryotic equivalents of the prokaryotic helicase. Although the positioning of the pseudoknot itself is clear, the mRNA passing through the entrance channel is too thin to be distinguished at the current resolution. Right: a comparison of the $80 \mathrm{~S}_{\mathrm{PK}}$ (top left) and the $80 \mathrm{~S}_{\mathrm{SL}}$ (top right) tRNAs (density in green; fitted atomic models in yellow). At the bottom a superposition of the two models is shown, showing the bending of the $80 \mathrm{~S}_{\mathrm{PK}}$ tRNA. 

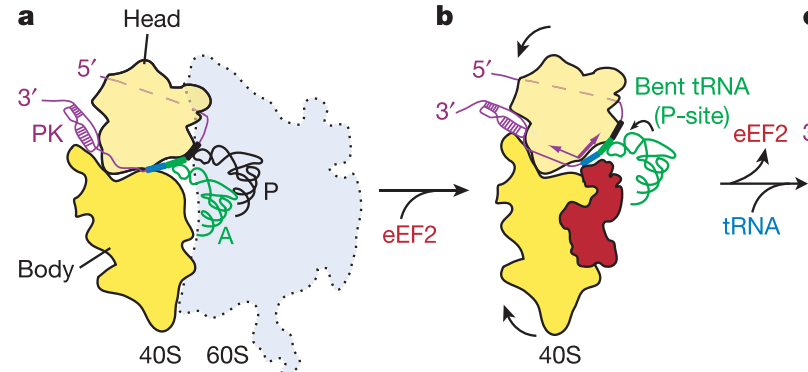

Figure 3 | A mechanical model for pseudoknot-induced -1 frameshifting. Three different states of the small subunit translating an mRNA containing a pseudoknot that induces -1 frameshifting are shown. a, The elongating ribosome approaching the pseudoknot in the zero reading frame. $\mathbf{b}$, Engagement with the pseudoknot, generating a frameshifting intermediate in which the small subunit is stalled during translocation with eEF2 bound, causing tension in the mRNA that bends the P-site tRNA in a (+) sense direction. As a result the anticodon-codon interaction breaks over the slippery sequence, allowing a spring-like relaxation of the tRNA in a (-) sense direction. c, Re-engagement of the tRNA with the mRNA, leaving the ribosome translating in the -1 reading frame.
( 5 ' direction), allowing the tRNA to re-pair with the mRNA in the -1 position. In support of this model, slippery sequences possessing a G-triplet or C-triplet in the A-site position show greatly reduced frameshifting ${ }^{5}$, which is explicable in terms of increased stability of the codon-anticodon complex and the consequent increased resistance to the mechanical forces applied. The mechanical model also offers an explanation for the capacity of RNAs of differing secondary structure to promote frameshifting to different extents ${ }^{2,12}$. The interaction between these RNA structures and the $80 \mathrm{~S}$ helicase is likely to be influenced both by conformational features and relative stability, so the amount of the strain on the tRNA and the likelihood of breakage of the codon-anticodon pair will depend on the secondary structure of the mRNA. For secondary structures that do not lead to frameshifting, including the SL used here, we propose that the helicase unwinds them in defined triplet steps coupled to normal translocation ${ }^{13}$, limiting mRNA tension. Such structures could still induce ribosomal pausing ${ }^{10,11}$, by virtue of their stability, but would be unable to generate the mRNA tension required for the distortion of tRNA and the induction of frameshifting during translocation.

\section{METHODS}

Detailed methods are given in Supplementary Methods.

Isolation of paused 80S mRNA complexes. Messenger RNAs (about 190 nucleotides) containing the minimal IBV pseudoknot ${ }^{23}$ or related stem-loop structure $^{23}$ were preannealed to a biotinylated RNA oligonucleotide and translated in rabbit reticulocyte lysates for $15 \mathrm{~min}$ at $27^{\circ} \mathrm{C}$ before the addition of cycloheximide. Ribosomes were pelleted through a sucrose cushion, resuspended and loaded on an avidin column. After extensive washing, mRNAribosome complexes were released by targeting a DNA oligonucleotide to a region downstream of the pseudoknot/stem-loop and adding RNase $\mathrm{H}$ (see Supplementary Fig. 1). Aliquots of ribosomes were taken and frozen at $-70^{\circ} \mathrm{C}$ before cryo-EM.

Cryo-EM, image processing and fitting of atomic structures. After particle picking and correction for contrast transfer function, the $80 \mathrm{~S}_{\mathrm{Apo}}$ data were used to generate a reconstruction $a b$ initio with the use of IMAGIC $^{24}$, refined iteratively in SPIDER ${ }^{25}$. The $80 \mathrm{~S}_{\mathrm{Apo}}$ map was used to align the $80 \mathrm{~S}_{\mathrm{PK}}$ or $80 \mathrm{~S}_{\mathrm{SL}}$ images, which were then also refined iteratively. Final maps were scaled in reciprocal space and sharpened by the application of a $B$ factor of $-500 \AA^{2}$. Fourier shell correlation ( 0.5 criterion) indicated the resolutions of the maps as follows: $80 \mathrm{~S}_{\mathrm{Apo}}, 14.0 \AA$; $80 \mathrm{~S}_{\mathrm{SL}}, 15.7 \AA ; 80 \mathrm{~S}_{\mathrm{PK}}, 16.2 \AA$. Atomic fitting was performed with $\mathrm{O}^{26}$ and $\mathrm{URO}^{27}$, making use of the published yeast atomic model ${ }^{15}$, and Protein Data Bank entries 1NOU and 2TRA. Figures were generated with BOBSCRIPT $^{28}$ and Raster3 ${ }^{29}$

\section{Received 3 February; accepted 20 March 2006.}

1. Ogle, J. M. \& Ramakrishnan, V. Structural insights into translational fidelity. Annu. Rev. Biochem. 74, 129-177 (2005).

2. Farabaugh, P. J. Programmed translational frameshifting. Microbiol. Rev. 60, 103-134 (1996).
3. Brierley, I. \& Dos Ramos, F. Ribosomal frameshifting in HIV-1 and the SARSCoV. Virus Res. advance online publication, 28 November 2005 (doi:10.1016/ j.virusres.2005.10.008)

4. Namy, O Rousset, J-P. Napthine, S. \& Brierley, I. Reprogrammed genetic decoding in cellular gene expression. Mol. Cell 13, 157-168 (2004).

5. Jacks, T., Madhani, H. D., Masiarz, F. R. \& Varmus, H. E. Signals for ribosomal frameshifting in the Rous sarcoma virus gag-pol region. Cell 55, 447-458 (1988).

6. Brierley, I., Digard, P. \& Inglis, S. C. Characterisation of an efficient coronavirus ribosomal frameshifting signal: Requirement for an RNA pseudoknot. Cell 57, 537-547 (1989).

7. Jacks, T. \& Varmus, H. E. Expression of the Rous sarcoma virus pol gene by ribosomal frameshifting. Science 230, 1237-1242 (1985).

8. Tu, C., Tzeng, T.-H. \& Bruenn, J. A. Ribosomal movement impeded at a pseudoknot required for frameshifting. Proc. Natl Acad. Sci. USA 89 , 8636-8640 (1992)

9. Lopinski, J. D., Dinman, J. D. \& Bruenn, J. A. Kinetics of ribosomal pausing during programmed -1 ribosomal frameshifting. Mol. Cell. Biol. 20, 1095-1103 (2000)

10. Kontos, H., Napthine, S. \& Brierley, I. Ribosomal pausing at a frameshifter RNA pseudoknot is sensitive to reading phase but shows little correlation with frameshift efficiency. Mol. Cell. Biol. 21, 8657-8670 (2001).

11. Somogyi, P., Jenner, A. J., Brierley, I. \& Inglis, S. C. Ribosomal pausing during translation of an RNA pseudoknot. Mol. Cell. Biol. 13, 6931-6940 (1993).

12. Atkins, J. F. et al. Overriding standard decoding: Implications of recoding for ribosome function and enrichment of gene expression. Cold Spring Harb. Symp. Quant. Biol. 66, 217-232 (2001).

13. Takyar, S., Hickerson, R. P. \& Noller, H. F. mRNA helicase activity of the ribosome. Cell 120, 49-58 (2005).

14. Yusupova, G. Z., Yusupov, M. M., Cate, J. H. \& Noller, H. F. The path of messenger RNA through the ribosome. Cell 106, 233-241 (2001).

15. Spahn, C. M. et al. Structure of the 80 S ribosome from Saccharomyces cerevisiae: tRNA-ribosome and subunit-subunit interactions. Cell 107, 373-386 (2001).

16. Frank, J. \& Agrawal, R. K. A ratchet-like inter-subunit reorganization of the ribosome during translocation. Nature 406, 318-322 (2000).

17. Spahn, C. M. et al. Domain movements of elongation factor eEF2 and the eukaryotic 80 S ribosome facilitate tRNA translocation. EMBO J. 23, 1008-1019 (2004).

18. Jorgensen, R. et al. Two crystal structures demonstrate large conformational changes in the eukaryotic ribosomal translocase. Nature Struct. Biol. 10, 379-385 (2003).

19. Weiss, R. B., Dunn, D. M., Shuh, M., Atkins, J. F. \& Gesteland, R. F. E. coli ribosomes re-phase on retroviral frameshift signals at rates ranging from 2 to 50 percent. New Biol. 1, 159-169 (1989).

20. Yelverton, E., Lindsley, D., Yamauchi, P. \& Gallant, J. A. The function of a ribosomal frameshifting signal from human immunodeficiency virus-1 in Escherichia coli. Mol. Microbiol. 11, 303-313 (1994).

21. Valle, M. et al. Incorporation of aminoacyl-tRNA into the ribosome as seen by cryo-electron microscopy. Nature Struct. Biol. 10, 899-906 (2003)

22. Plant, E. P. et al. The $9 \mathrm{~A}$ solution: How mRNA pseudoknots promote efficient programmed -1 ribosomal frameshifting. RNA 9, 168-174 (2003).

23. Brierley, I., Jenner, A. J. \& Inglis, S. C. Mutational analysis of the 'slippery sequence' component of a coronavirus ribosomal frameshifting signal. J. Mol. Biol. 227, 463-479 (1992).

24. van Heel, M., Harauz, G., Orlova, E. V., Schmidt, R. \& Schatz, M. A new generation of the IMAGIC image processing system. J. Struct. Biol. 116, 17-24 (1996).

25. Frank, J. et al. SPIDER and WEB: processing and visualization of images in 3D 
electron microscopy and related fields. J. Struct. Biol. 116, 190-199 (1996).

26. Jones, T. A., Zou, J. Y., Cowan, S. W. \& Kjeldgaard, M. Improved methods for building protein models in electron density maps and the location of errors in these models. Acta Crystallogr. A 47, 110-119 (1991).

27. Navaza, J., Lepault, J., Rey, F. A., Alvarez-Rua, C. \& Borge, J. On the fitting of model electron densities into EM reconstructions: a reciprocal-space formulation. Acta Crystallogr. D 58, 1820-1825 (2002).

28. Esnouf, R. M. Further additions to MolScript version 1.4, including reading and contouring of electron-density maps. Acta Crystallogr. D 55, 938-940 (1999).

29. Merritt, E. A. \& Murphy, M. E. Raster3D Version 2.0. A program for photorealistic molecular graphics. Acta Crystallogr. D 50, 869-873 (1994).

Supplementary Information is linked to the online version of the paper at www.nature.com/nature.

Acknowledgements I.B. thanks P. Farabaugh for helpful discussions. This work was supported by grants from the Biotechnology and Biological Sciences
Research Council, UK, and the Medical Research Council, UK. S.J.M. holds a Wellcome Trust Structural Biology Studentship, D.I.S. is an MRC Research Professor, and R.J.C.G. is a Royal Society University Research Fellow.

Author Contributions O.N. and S.J.M. contributed equally to this work. O.N. and I.B. purified the stalled ribosome complexes; S.J.M., D.I.S. and R.J.C.G. solved the structures. All authors discussed the results and contributed to writing of the manuscript.

Author Information Electron-density maps have been deposited in the European Bioinformatics Institute Electron Microscopy database, accession numbers EMD-1197, EMD-1198 and EMD-1199 (www.ebi.ac.uk/msd/iims/ 3D_EMdep.html). Reprints and permissions information is available at npg.nature.com/reprintsandpermissions. The authors declare no competing financial interests. Correspondence and requests for materials should be addressed to I.B. (ib103@mole.bio.cam.ac.uk) or R.J.C.G. (gilbert@strubi.ox.ac.uk). 